\title{
Responsabilidad social, factor integrador en universidades públicas
}

\author{
Integrator social responsibility factor \\ in public universities
}

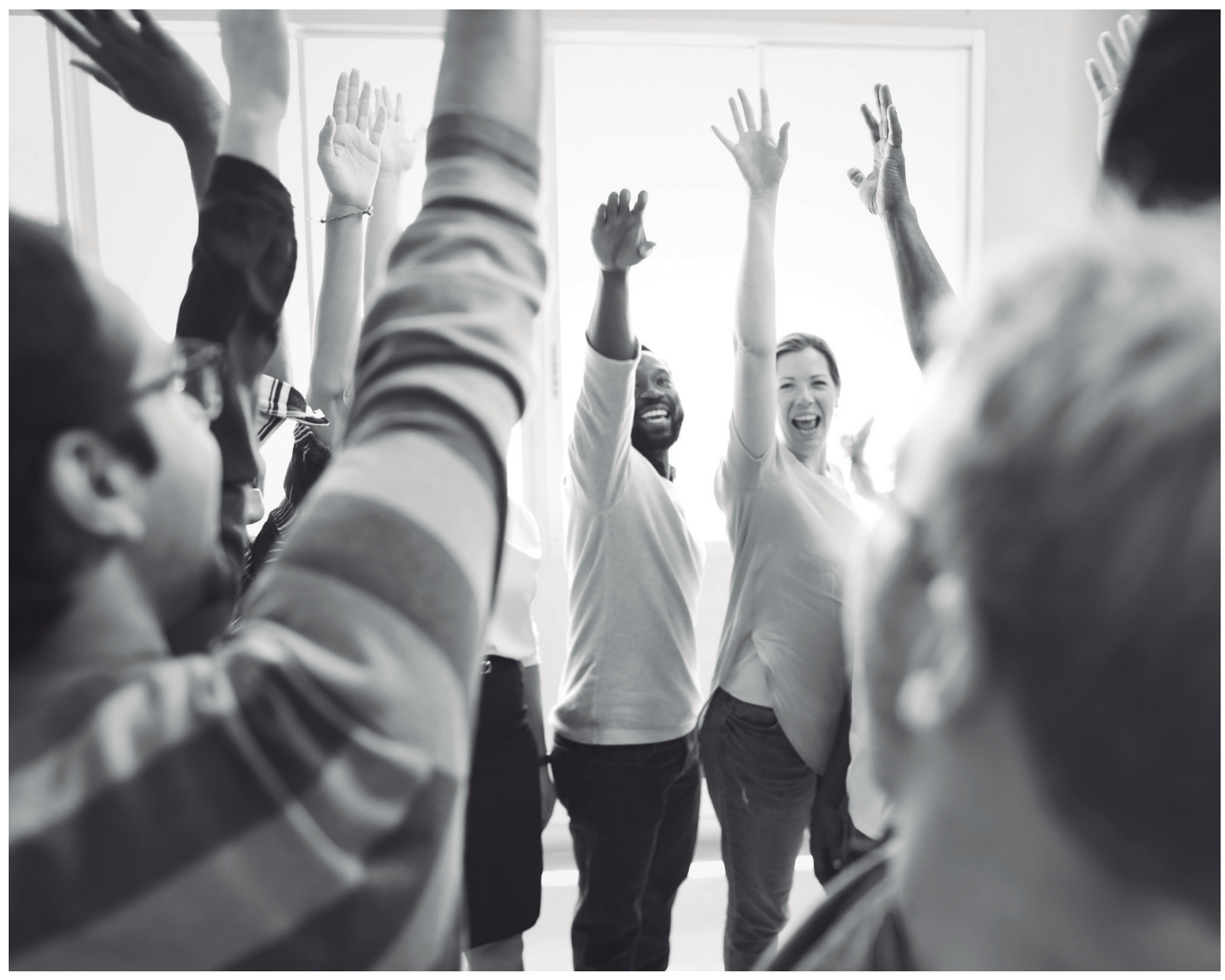




\title{
Responsabilidad social, factor integrador en universidades públicas ${ }^{1}$
}

\section{Integrator social responsibility factor in public universities}

\author{
Annherys Isabel Paz Marcano, ${ }^{2}$ José Antonio Sánchez González, ${ }^{3}$ \\ Iván José Sánchez Valbuena ${ }^{4}$
}

Artículo recibido en agosto de 2017; artículo aceptado en marzo de 2018.

Este artículo puede compartirse bajo la Licencia Creative Commons Atribución-No Comercial-Compartir lgual 2.0 Genérica y

se referencia usando el siguiente formato: Paz, A., Sánchez, J. \& Sánchez, I. (2018). Responsabilidad social factor integrador en

universidades públicas. I+D Revista de Investigaciones, 12 (2), 39-50. DOI: https://doi.org/10.33304/revinv.v12n2-2018004

\section{Resumen}

El presente artículo tiene como propósito analizar la responsabilidad social como factor integrador en universidades públicas. Metodológicamente comprende un enfoque epistemológico positivista, el tipo de estudio es analítico, descriptivo, con diseño no experimental, transaccional, de campo. La población objeto está conformada por diecinueve decanos de las universidades públicas del estado Zulia, en Venezuela. Para la recolección de información se empleó la técnica de la encuesta, mientras el instrumento es un cuestionario de diez ítems con opciones de respuestas múltiples, validado por diez expertos. La confiabilidad se calculó con la fórmula de Alfa Cronbach, que arrojó un valor de 0,94 ; los resultados se analizaron usando la estadística descriptiva. A manera de conclusión se observó que las universidades públicas ejercen un compromiso socialmente responsable, cuya actuación se constituye en un factor integrador de la ética, el trabajo y el liderazgo entre los colaboradores para dinamizar la excelencia y la calidad en la gestión institucional desde una visión holística, tanto de manera interna como externa a ella.

Palabras clave: responsabilidad social, factor integrador, universidades públicas.

1. Artículo de Investigación, de tipo analítico-descriptivo, con diseño no experimental. Resultado de la investigación culminado, titulado Responsabilidad social en universidades. Desarrollado en la Universidad de La Guajira. Km.5 Vía Maicao - Tel. (57) (5) 7282729 - Riohacha, La Guajira (Colombia). Inició en septiembre de 2014 y finalizó en diciembre de 2016.

2. Doctora en Ciencias Gerenciales (Universidad Dr. Rafael Belloso Chacín URBE-Venezuela). Postdoctora en Gerencia de las Organizaciones (Universidad Dr. Rafael Belloso Chacín URBE-Venezuela). Postdoctora en Integración y Desarrollo en América Latina (Universidad Dr. Rafael Belloso Chacín. URBE-Venezuela). Magíster en Gerencia de Recursos Humanos. (Universidad Dr. Rafael Belloso Chacín. URBE-Venezuela). Licenciada en Administración de Empresas (Universidad Dr. Rafael Belloso Chacín URBE-Venezuela). Docente e investigadora de la Universidad de La Guajira- Colombia. Grupo de Investigación Aika, Categoría A. Investigador Senior de Colciencias. ORCID ID: https://orcid.org/0000-0001-7538-1563. Correo electrónico: aipaz@uniguajira.edu.co; annheryspaz@hotmail.com.

3. Doctor en Ciencias Gerenciales (Universidad Dr. Rafael Belloso Chacín URBE-Venezuela). Postdoctor en Ciencias Humanas (Luz-Venezuela). Magíster en Gerencia Empresarial (Universidad Dr. Rafael Belloso Chacín URBE-Venezuela). Especialista en Alta Gerencia (Universidad Autónoma del Caribe). Especialista en Planeación Educativa (Pontificia-Universidad Javeriana). Licenciado en Administración Hotelera y Turismo (Universidad Autónoma del Caribe). Docente e investigador de la Universidad de La Guajira- Colombia. Grupo de Investigación Aika, Categoría A. Investigador Senior de Colciencias. ORCID ID: https://orcid.org/0000-0001-62723851. Correo electrónico institucional: jsanchez@uniguajira.edu.co.

4. Licenciado en Administración (Universidad de La Guajira, Uniguajira Colombia) Msc. en Gerencia empresarial. (Universidad Dr. Rafael Belloso Chacín URBE-Venezuela). Docente e investigador de la Universidad de la Guajira-Colombia. Grupo de Investigación: Aika, Categoría A. Investigador Junior de Colciencias ORCID ID: https://orcid.org/0000-0002-3134-3851. Correo electrónico institucional: ijsanchez@uniguajira.edu.co. 


\begin{abstract}
This article aims to analyze the social responsibility as an integrating factor in public universities. Methodologically it comprises a positivist epistemological approach, type of analytical study, descriptive, not experimental, transactional, field design, population 19 deans of public universities in the state Zulia Venezuela, using data collection the survey technique, while the 10-item questionnaire with multiple response options, validated by (10) experts and calculated reliability with Cronbach al pha formula, reflecting a value of 0,94 instrument; the results were analyzed using descriptive statistics. In conclusion, it was found that public universities, have a socially responsible commitment, represented his performance an integrating factor of ethics, work and leadership among employees to boost excellence and quality in institutional management from a holistic view both internally and external to it.
\end{abstract}

Keywords: social responsibility; integrating factor; public universities.

\section{Introducción}

La globalización es un proceso creciente a escala mundial en el campo de los negocios, que no excluye la gestión de las universidades, lo que conlleva la inclusión de nuevas tendencias en el plan de acción que las convierte en un medio participativo e integrador de la sociedad mediante el desarrollo de actividades estratégicas que agregan valor a la base de la actuación académica, investigativa y de extensión, donde, en conjunto, se establecen objetivos para alcanzar la competitividad institucional con apoyo de los grupos de interés.

Por ello, para afrontar las exigencias del entorno las universidades públicas fomentan cambios en la estructura de las actividades académicas, investigativas, tecnológicas, sociales, culturales, económicas y ecológicas, entre otras, que por sí solas no podrían llevarse a cabo si no se involucra a los colaboradores en el aporte de ideas para la actualización y establecimiento de objetivos estratégicos que guían la excelencia en la calidad de la gestión institucional. Por tanto, comprende las directrices fundadas en el compromiso y la actuación ética de toda la comunidad universitaria con el propósito de integrarse con el entorno.

Paz, Núñez, Salóm y Rosales (2013) destacan que el propósito educativo no solo debe fundamentarse en los pilares de la gestión universitaria como son la docencia, la investigación y la extensión, sino que debe ir más allá, centrándose en un sentido humanístico mediante un liderazgo académico basado en las fortalezas éticomorales del colectivo, como parte de su responsabilidad en la formación de una sociedad con rostro humano, social y moral transmisible a todos los grupos de interés, en tanto que es parte integral de dicha sociedad.

De tal manera que para contraponer las dificultades y aprovechar las oportunidades, las instituciones se convierten en un factor integrador de la comunidad con su entorno, mediante una actuación basada en unos principios éticos que determinan la responsabilidad social de las actividades de trabajo en el ejercicio de su voluntariedad para obtener un desempeño social transparente. Para cumplir con su gestión y cimentar universidades sostenibles, se requiere de colaboradores con competencias integrales basadas en la ética, el trabajo y el liderazgo, que desarrollen sus actividades con un sentido humanizado de corresponsabilidad en el ejercicio de sus funciones, pero conjuntamente creando un sentido responsable cónsonos con las realidades del hoy con visión prospectiva hacia el mañana.

Según Global University Network for Education (2009), la intención y la direccionalidad de las funciones universitarias concretan la pertinencia como expresión de responsabilidad, para obrar de acuerdo con los requerimientos, dinámica y evolución de la sociedad actual, siendo así como la universidad debe ser consciente de que se está gestando una nueva sociedad. Cabe señalar que pese a que la responsabilidad social no es un tema novedoso en el ámbito universitario, en las últimas décadas se ha convertido en un factor de referencia para la integración de la gestión institucional con grupos de interés tanto internos como externos.

Este hecho le ha permitido a la universidad incursionar en la recuperación de espacios sociales mediante un compromiso ético compartido entre los colaboradores en alianzas con empresas públicas, privadas y organizaciones sin fines de lucro (ONG), que, de forma recíproca proponen políticas a fin de satisfacer las necesidades del colectivo social, atendiendo a sus problemas y asumiendo la medición de los indicadores del desempeño social responsable ante los grupos de interés (Serrano, 2015).

Para Paz, Sánchez y Magdaniel (2016), la responsabilidad social es un compromiso de todos que debe plantearse como un modelo de gestión compartido entre las empresas y la sociedad, y viceversa, que privilegie la calidad de vida de las personas para entramar un tejido social integrador; es decir, no solo velar por el alcance de los objetivos en términos económicos sino ético-morales 
a fin de promover la convivencia justa en la sociedad para el bienestar del colectivo humano.

Según esta concepción, se infiere que mediante la responsabilidad social se proyecta la integración doble acción universidad-sociedad y viceversa, pudiendo fortalecer el bienestar social con iniciativas sostenibles, $y$, a la vez, generar ventajas competitivas mediante estrategias universitarias que conlleven la práctica de acciones responsables y con ello a la evaluación de los factores insertos en su gestión, para establecer sinergias con planes que generen compromisos integrales, donde se involucre la universidad-colaboradores-sociedad y viceversa, dando como resultado una proyección, imagen y reputación de academia con sentido social. Por tanto, las universidades tienen la necesidad de repensar su posición y función en la sociedad de acuerdo al análisis de los cambios económicos, sociales, políticos y culturales esbozados en las tendencias que marcan los objetivos del milenio.

Por ello, la responsabilidad social en la universidad pública comprende un proceso de apoyo donde se proyecta la práctica de acciones responsables destinadas a su gente y al entorno desde un enfoque ético, enlazado en el vínculo estratégico del compromiso institucional con los grupos de interés mediante la actuación moral para cultivar un equilibrio entre las partes involucradas; enmarcado su comportamiento en la filosófica de gestión y valores éticos, el cual ubica a los colaboradores como centro de participación ciudadana significativa en la dimensión social desde una visión holística (García \& González, 2015).

Para Paz, Harris y Molero (2010) la responsabilidad social universitaria va más allá de un desempeño organizacional, pues debe develar el comportamiento personal de los miembros adscritos a la universidad, pues no se trata de una conducta inexcusable para dar valor social al proceso irreversible de los cambios del entorno, sino, por el contrario, de crear un código ético valorativo del crecimiento personal y profesional del ser humano mediante la práctica de acciones sociales responsables.

De tal manera que la responsabilidad social adquiere relevancia en el compromiso institucional con sus colaboradores, representando desde sus factores integradores de la ética, trabajo y liderazgo indicadores que dinamizan el alcance de los objetivos, exponiendo sus conocimientos e ideas, pero además promoviendo el quehacer de sus responsabilidades, generando un esfuerzo que activa la actuación de los grupos de interés a favor de la excelencia y proyección social suscrita en la base de la gestión universitaria. A partir de los aspectos expuestos se plantea como objetivo analizar la responsabilidad social como factor integrador en universidades públicas. De esta manera, la investigación aborda aspectos contentivos a la fundamentación teórica mediante la conceptualización de la responsabilidad social seguidamente de los factores de la responsabilidad social, tales como ética, trabajo y liderazgo.

\section{Responsabilidad social}

Según Sánchez (2008), citado por Paz, Sánchez y Magdaniel (2016), la responsabilidad social comprende las estrategias de la gestión de las empresas, orientadas a fomentar las relaciones de trabajo, la competitividad y la cohesión social, que desde la voluntariedad la hacen ser reconocida como empresas socialmente responsables. De acuerdo con Pérez (2009), la aplicación de la responsabilidad social universitaria es un factor de diferenciación de la universidad en que se aplica, por tanto, de éxito en un entorno cada vez más competitivo.

Tal como lo proponen Paz, Paz y Franco (2013), la responsabilidad social permite alcanzar ventajas competitivas y construir un balance ético organizacional. Por otra parte, Pelekais y Aguirre (2008) afirman que la responsabilidad social tiene su fundamento en el interior de la organización, la cual asume los valores y principios reguladores de la plataforma filosófica a través de la internalización de éstos, exteriorizándose en el lugar donde se encuentra asentada dicha organización.

En líneas generales, la responsabilidad social comprende la acción voluntaria ejercida como cimiento de la gestión estratégica de las universidades, cuya integración puede visualizarse como el compromiso de los grupos de interés involucrados en las actividades para alcanzar la excelencia institucional. Esto se logra mediante la ejecución de las actividades en la sociedad, pero también haciendo visible la reciprocidad de las comunidades con la institución; es decir, se basa en la participación activa de ambos agentes por el bien común. Vallaeys (2003), citado en Núñez, Salom, Rosales y Paz (2012), propone que la responsabilidad social universitaria articule, a partir de una visión holística, las diferentes partes de la institución en un proyecto de promoción social de principios éticos y de desarrollo social equitativo pero sostenible, para la formación de profesionales como ciudadanos responsables y la producción y transmisión de'saberes', asimismo responsables.

Desde la posición de Pérez (2009), la responsabilidad social inserta en los escenarios universitarios es una nueva manera de funcionamiento de la universidad, un nuevo modo de hacer y comportarse basado en una relación más fluida y directa de ésta y con su entorno 
social, para lo que se toman en consideración los efectos, repercusiones y expectativas que la actividad de la universidad genera, tanto en sus propios miembros (docentes, investigadores, personal de administración y estudiantes), como en la sociedad.

\section{Factores de la responsabilidad social}

Gallego (2006) propone que mirar las organizaciones desde un enfoque sistémico permite verlas como un conjunto de partes interactuantes e interdependientes que, en su dinámica, conforman un sistema mayor que es la sociedad. Desde esta perspectiva, puede comprenderse que cada organización afecta y es afectada por el entorno y que esta mutua relación de beneficio o perjuicio determina, en un alto grado, su supervivencia, siendo una forma de entender lo que hoy se conoce como responsabilidad social. De acuerdo con Paz,
Sánchez y Magdaniel (2016), la responsabilidad social se vincula con diversos factores del entorno tanto interno como externo a la empresa, considerando su influencia en apoyo a la realidad en la cual se desenvuelve.

En consecuencia, una empresa se considera responsable socialmente cuando incluye entre sus principios de gestión la ética, la moral y principios tanto cívicos como ciudadanos, al mismo tiempo que valores discriminados según los intereses de la institución. Por su parte, Guédez (2008) expresa que los factores que apoyan el hecho de que los negocios sean socialmente responsables son ética, trabajo y liderazgo (ver Figura 1).

\section{Ética}

Para Guédez (2006) la ética se aplica en el ámbito de la responsabilidad social, donde se muestra el compromiso

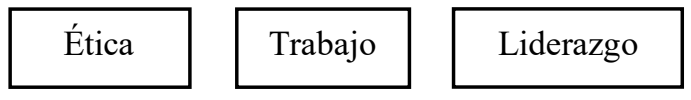

Figura 1. Factores de la responsabilidad social. Fuente: Adaptado de Guédez (2008)

con la comunidad, ya que no existe una responsabilidad social sin ética, puesto que ésta se desarrolla sin perjudicar a otras personas o instituciones. Por consiguiente, un comportamiento ético no solo radica en evitar la corrupción, sino en respetar las libertades, los anhelos y las potencialidades de los demás.

Velásquez (2008) afirma que la ética es la actividad de examinar los estándares morales de uno mismo o los estándares morales de la sociedad, y preguntarse cómo se aplican a la vida y si son razonables o no. Esto es, si se hallan sustentados por buenas o malas razones. En consecuencia, una persona empieza a ser ética cuando toma los estándares morales asimilados de la familia, la iglesia y los amigos.

Por su parte, Soto y Cárdenas (2007) sostienen que la ética es el estudio sistemático de la naturaleza de los conceptos axiológicos, como bien, mal, equivocado, y de los principios generales que justifican la aplicación de ellos a alguna acción o acto; su importancia radica en la relación con las nociones fundamentales de moralidad y éstas pueden tener consecuencias en relación con la conducta de las personas. La ética comprende el principio de actuación transparente de las universidades públicas, y conlleva, desde sus directrices, el desempeño ético de los colaboradores en el ejercicio de sus funciones administrativas, académicas, investigativas y de extensión.

\section{Trabajo}

Guédez (2008) postuló que el término trabajo es una actividad propia del ser humano, si bien otros seres también actúan dirigiendo sus energías coordinadamente, con una finalidad determinada. Sin embargo, el trabajo como proceso entre la naturaleza y el hombre es exclusivamente humano, mientras, en un sentido amplio, es toda actividad humana que transforma la naturaleza a partir de cierta materia dada. Para Fernández (2009) el trabajo debería ser ofrecido y aceptado con libertad, pudiendo ser abandonado por los empleados de acuerdo con las reglas establecidas, mostrando oportunidades y trato equitativo en cualquier actividad relacionada con este. Según Maslow, citado por Ramos (2006), el trabajo es un paso para lograr la realización personal y un motivo para vivir, convirtiéndose en un hecho armónico donde se conjuga la existencia con el trabajo y la satisfacción, hecho que demanda la capacidad de sensibilidad para vivir esos sentimientos.

Por su parte, Ramos agrega que el trabajo busca la realización personal, traducido de la recompensa del quehacer laboral, pero cultivando el alcance de objetivos organizacionales, teniendo esté una dimensión natural, de servicio, ética, trascedente y social; en este contexto, la dimensión social del trabajo es la contribución al bienestar de la sociedad a la que pertenece, dejando de ser una acción individual para convertirse en colectiva, 
que implica la práctica de valores concretos. De tal manera que el trabajo en las universidades públicas comprende el resultado del aporte de conocimientos e ideas del hombre, puesto de manifiesto en el desempeño de sus funciones, basado en los principios éticos que regulan su comportamiento como persona integral ante la sociedad, siendo creativos e innovadores en el ejercicio de las responsabilidades asignadas en el cargo ocupado.

\section{Liderazgo}

Guédez (2008) afirma que el liderazgo juega un papel esencial en cada una de las dinámicas organizacionales, siendo un líder resonante que crea más, llegando así a una conclusión determinante: su auténtico desarrollo debe asentarse en una visión holística, que va más allá de una nueva planificación de carrera profesional, teniendo en cuenta las riquezas de la vida humana. Por otra parte, Lussier y Achua (2008) definen el liderazgo como el proceso de influencia de líderes y seguidores para alcanzar los objetivos de la organización mediante el cambio. Perozo y Paz (2016) agregan que las habilidades y características del líder lo identifican como un ser humano genuino, cuya actuación en el entorno es un campo dinamizador para el desarrollo de las actividades, en todo caso potenciando su comportamiento, pero transmitiendo sus conocimientos a otros colaboradores para que, de manera integral, activen las competencias como equipo de trabajo, que ofrece resultados satisfactorios en los objetivos planteados.

De igual manera, Brittel (2007) explica que el liderazgo es la habilidad que determinadas personas tienen de influir en otras para guiarlas en la realización de su trabajo y hacia la obtención de logros significativos. Para Robbins (2009) el liderazgo es la capacidad de una persona para influir en un grupo para alcanzar metas, es decir, desempeña un papel en el comportamiento de grupos relacionado con la motivación, pues al entender esta última, se tiene una mejor visión del deseo de las personas y sus comportamientos. Según Chiavenato (2011) el líder tiene la capacidad de inspirar y guiar a individuos o grupos; asimismo comprende la influencia interpersonal ejercida en una situación dirigida, a través del proceso de comunicación humana, a la consecución de uno o diversos objetivos específicos.

En líneas generales, desarrollar actividades de responsabilidad social en las universidades públicas implica guiarse por factores integradores como la ética, el trabajo y el liderazgo, que, en conjunto, se asientan en un enfoque transparente que da lugar a la práctica responsable de actividades objetivas, pero simultáneamente con el trabajo de otorgar respuesta a una necesidad o problema del entorno tanto interno como externo. En este sentido, es protagonista de un liderazgo social que crea reputación y credibilidad entre los grupos de interés haciendo de la academia un medio de proyección social sostenible mediante la formación de hombres como ente social sustentado en valores y principios éticos morales.

De acuerdo con Villalobos (2008), citado por Núñez et al. (2012), para los líderes es un desafío formar profesionales cuyos valores respondan a la actuación responsable y comprometida con la solución de necesidades y problemáticas de su entorno laboral y social inmediato, centrando su propósito educativo en la idea del liderazgo académico basado en fortalezas morales.

\section{Método}

El presente artículo se esboza metodológicamente desde el quehacer científico mediante el desarrollo de actividades investigativas para recolectar y analizar información referente al tema estudiado, estableciendo criterios interpretativos para otorgar respuestas objetivas, válidas y confiables. Según Hernández, Fernández y Baptista (2014), la recolección de datos implica elaborar un plan detallado de procedimientos que conducen y reúnen datos con un propósito.

\section{Enfoque epistemológico}

La investigación se apoyó en técnicas metodológicas de naturaleza cuantitativa, permitiendo la aplicación de instrumentos estadísticos para conocer la opinión de los encuestados e interpretar los datos en frecuencias y estimaciones sobre la variable responsabilidad social en las universidades públicas. El objetivo de investigación, referente analizar la responsabilidad social como factor integrador en universidades públicas, se suscribe en el paradigma científico positivista, el cual expresa que fuera del ser humano no existe una realidad social externa y objetiva, sino que se busca indagar en hechos reales, utilizando herramientas metodológicas para estudiar la variable de estudio.

Chávez (2007) afirma que la corriente positivista se manifiesta acerca de la verdad sin modificarla, aplicando el método cuantitativo, por lo que se estudian variables sobre la base de la cuantificación como un conjunto de reglas previamente establecidas, relacionadas en parte con las operaciones empíricas concretas de técnicas estadísticas descriptivas, paramétricas o no.

De acuerdo con Guba (1989), citado por Guanipa (2010), la base filosófica del investigador positivista 
o cuantitativo se ocupa de los hechos y causas de los fenómenos sociales, desinteresándose de los estados subjetivos de las personas, tendiendo a ver el mundo bajo una perspectiva causal, determinista y predictiva, tratando de reducir la realidad imponiéndole condiciones y antecedentes prefabricados con una postura estructurada, centrada, singular.

\section{Tipo de investigación}

En cuanto a los objetivos, la investigación es de tipo analítico y descriptivo; el primero interpreta características acerca de la variable responsabilidad social a partir de los factores integradores en las universidades públicas, mientras el segundo, es decir, lo descriptivo, alude a orientar la acepción de información compilada asociada a la variable objeto de estudio.

Para Hurtado (2008) la investigación analítica reinterpreta criterios dependientes de los objetivos en estudio. Por otro lado, Hernández, Fernández Baptista (2014) afirman que la investigación descriptiva busca especificar propiedades, características y rasgos importantes del fenómeno analizado, describiendo tendencias de un grupo o población, partiendo de perfiles de personas, grupos, comunidades, procesos u objetos o cualquier otro fenómeno sometido a análisis. Este tipo de investigación facilita desarrollar aspectos en relación con el objeto de investigación, logrando analizar los datos obtenidos acerca de la variable de estudio, es decir la responsabilidad social como factor integrador en universidades públicas.

En relación con el diseño, la investigación se suscribe al no experimental, transaccional de campo, en el cual los investigadores no manipulan la información sino que compilan datos en fuentes reales durante un lapso planificado. Según Hernández et al., los diseños no experimentales de estudios transversales recolectan datos en un solo momento, en un tiempo único, teniendo como propósito describir variables y analizar su incidencia e interrelación en un momento dado. No obstante, los diseños transaccionales descriptivos indagan la incidencia de las modalidades o niveles de una o más variables en una población.

Por su parte, Arias (2012) sostiene que la investigación de campo recolecta datos directamente en la realidad donde se generan los hechos, sin manipular o controlar variable alguna. Tamayo (2004) aporta un diseño de campo en el que los datos se recogen directamente de la realidad, por lo cual se les denomina primarios. En la investigación, la indagación de la información concerniente a la variable responsabilidad social como factor integrador en universidades públicas se compiló a partir de la opinión emitida por los decanos de las universidades públicas del estado Zulia, Venezuela.

\section{Participantes}

Hernández, Fernández y Baptista (2014) establecen que la población es el conjunto de todos los casos que concuerdan con determinadas especificaciones, partiendo de características de contenido, de lugar y de tiempo, con la finalidad de establecer los parámetros muestrales, en los que se incluye la totalidad de los sujetos, objetos, fenómenos, documentos o situaciones que se desee investigar.

La población de estudio está conformada por diecinueve decanos de las universidades públicas del estado Zulia, Venezuela, y se caracteriza como accesible y finita, razón por la que se analizó la información suministrada por la totalidad de los sujetos, convirtiéndose en censo. Méndez (2012) sostiene que un censo estudia todos los elementos involucrados en la población, siendo aconsejable aplicarlo cuando esta es finita; asimismo, cuando se requiere información inmediata del estudio.

\section{Materiales e instrumentos}

Encuesta. Para la compilación de información de interés sobre la variable factores de la responsabilidad social se consideró pertinente aplicar la técnica de observación por encuesta, definida por Tamayo (2004) como aquella que permite la observación directa, orientada a la percepción de hechos o fenómenos de interés en la investigación, valiéndose de instrumentos para tales fines. Para Méndez (2012) la encuesta es un instrumento de observación formado por una serie de preguntas formuladas, cuyas respuestas son anotadas por el encuestador; esta permite conocer las motivaciones, actitudes y opiniones de los individuos en relación con el objeto de investigación.

Instrumento. Sobre los aspectos suscritos, resulta esencial aplicar instrumentos como medio de recolección de datos asociados a la variable investigada, permitiendo la medición a través de herramientas científicas como el cuestionario, un recurso empleado por los investigadores para recabar información del objeto de estudio. Para Hernández, Fernández y Baptista (2014) los instrumentos de medición son recursos utilizados por el investigador para registrar información o datos sobre las variables que tiene en mente; su función de medición es establecer una correspondencia entre el mundo real y el conceptual, es decir, encontrar sentido a ese segmento real que se trata de describir. 
El cuestionario, que se estructuró sobre la base del contexto teórico de la variable responsabilidad social como factor integrador, está conformado por un conjunto de diez ítems de respuesta múltiple de siempre, casi siempre, a veces, casi nunca y nunca, orientados a medir las dimensiones e indicadores que determinan el comportamiento de la variable en el escenario investigativo, en este particular, el sector universitario público. Hernández et al., explican que un cuestionario, que consiste en un conjunto de preguntas respecto de una o más variables a medir, es el instrumento más utilizado para recolectar datos.

Vale acotar que el referido instrumento fue previamente validado por diez expertos y su confiabilidad se calculó por fórmula de Alfa Cronbach, alcanzando un resultado de 0,94 , representado porcentualmente por un $94 \%$ de fiabilidad; los resultados se interpretaron usando la estadística descriptiva.

\section{Procedimiento}

El trabajo parte del anteproyecto de investigación, en el que se enuncian el planteamiento, la formulación del problema, los objetivos y justificación, al tiempo que se delimita la investigación. Seguidamente, se establecen los antecedentes, las bases teóricas, así como el sistema y la operacionalización de la variable estudiada.

Posteriormente, se construyó el marco metodológico, agregando el enfoque epistemológico, y el tipo y diseño de la investigación. En cuanto a las unidades informantes se seleccionó como población a diecinueve decanos de las facultades que integran las universidades públicas del estado Zulia, Venezuela, por ser los encargados de promover las actividades de responsabilidad social. Debido a sus características finitas y accesibles no se realizó cálculo muestral, sino que se estudió en su totalidad, caracterizándose como censo poblacional.

Para compilar la información se aplicaron técnicas e instrumentos de recolección de datos, siendo el primero la encuesta y el segundo, un cuestionario. La información se abordó mediante el tratamiento estadístico descriptivo, que consiste en calcular la distribución de frecuencias absolutas y relativas. Asimismo, se aplicaron medidas de tendencia central, tales como media aritmética, geométrica, mediana y moda. Las medidas de dispersión, varianza, desviación estándar, coeficiente de variación, entre otros, se analizaron con el software hoja de cálculo de Excel, para cuantificar, organizar, registrar y tabular los datos, a fin de analizar, interpretar y graficar los resultados.

Finalmente, se efectuó el análisis y discusión de los resultados considerando las teorías consultadas, conclusiones, recomendaciones, así como las referencias bibliográficas que soportan el trabajo de investigación. Concluido el desarrollo de los aspectos planteados, se procedió a elaborar el informe final, así como el presente artículo, producto del trabajo realizado.

\section{Resultados}

A continuación se presenta el análisis de las respuestas obtenidas de la población objeto de estudio posterior a la aplicación del cuestionario como instrumento compilador de datos de la variable responsabilidad social factor integrador en las universidades públicas. Para ello se realizó un análisis estadístico basado en el ordenamiento descriptivo de frecuencias absolutas y relativas, de porcentajes promedio por categoría, de los indicadores y las dimensiones; también se calcularon las medidas de tendencia central como la media aritmética para datos no agrupados $u$ ordenados, que fueron interpretados siguiendo el siguiente baremo (ver Tabla 1).

De acuerdo con la Tabla 2 y la Figura 2, los resultados para el ítem 1 del indicador Ética muestran que el $63 \%$ de la población encuestada dijo que las universidades públicas siempre desarrollan una dinámica institucional reflejada en la actuación de principios éticos; un $32 \%$ dijo que casi siempre y un $5 \%$, que a veces. Por otro lado, la información

Tabla 1

Baremo de interpretación del promedio de datos

\begin{tabular}{cc}
\hline Rangos & Categorías \\
\hline $1,00 \geq 1,80$ & Muy baja \\
$1,81 \geq 2,60$ & Baja \\
$2,61 \geq 3,40$ & Moderada \\
$3,41 \geq 4,20$ & Alta \\
$4,21 \geq 5,00$ & Muy alta \\
\hline
\end{tabular}

Fuente: Tomado de Paz, Sánchez, Magdaniel y Robles (2017). 
Tabla 2

Factores de la responsabilidad social

\begin{tabular}{|c|c|c|c|c|c|c|c|c|c|c|c|c|c|}
\hline \multirow{3}{*}{ Indicadores } & \multirow{3}{*}{ Ítems } & \multicolumn{10}{|c|}{ Alternativas de respuesta } & \multicolumn{2}{|c|}{ Promedios } \\
\hline & & \multicolumn{2}{|c|}{ Siempre } & \multicolumn{2}{|c|}{$\begin{array}{c}\text { Casi } \\
\text { siempre }\end{array}$} & \multicolumn{2}{|c|}{$\begin{array}{c}\text { A } \\
\text { veces }\end{array}$} & \multicolumn{2}{|c|}{$\begin{array}{c}\text { Casi } \\
\text { nunca }\end{array}$} & \multicolumn{2}{|c|}{ Nunca } & \multirow{2}{*}{$\begin{array}{c}\text { Promedio } \\
\text { Ítems }\end{array}$} & \multirow[t]{2}{*}{$\begin{array}{l}\text { Promedio } \\
\text { Indicadores }\end{array}$} \\
\hline & & $\mathbf{F a}$ & Fr\% & $\mathbf{F a}$ & Fr\% & $\mathbf{F a}$ & Fr\% & $\mathbf{F a}$ & Fr\% & $\mathbf{F a}$ & $\mathrm{Fr} \%$ & & \\
\hline \multirow{4}{*}{ Ética } & 1 & 12 & $63 \%$ & 6 & $32 \%$ & 1 & $5 \%$ & 0 & $0 \%$ & 0 & $0 \%$ & 4,58 & \multirow{4}{*}{4,65} \\
\hline & 2 & 15 & $79 \%$ & 3 & $16 \%$ & 1 & $5 \%$ & 0 & $0 \%$ & 0 & $0 \%$ & 4,74 & \\
\hline & 3 & 14 & $74 \%$ & 4 & $21 \%$ & 0 & $0 \%$ & 1 & $5 \%$ & 1 & $5 \%$ & 4,63 & \\
\hline & 4 & 11 & $58 \%$ & 6 & $32 \%$ & 3 & $10 \%$ & 0 & $0 \%$ & 0 & $0 \%$ & 4,47 & \\
\hline \multirow{4}{*}{ Trabajo } & 5 & 12 & $63 \%$ & 6 & $32 \%$ & 1 & $5 \%$ & 0 & $0 \%$ & 0 & $0 \%$ & 4,58 & \multirow{4}{*}{4,60} \\
\hline & 6 & 15 & $79 \%$ & 3 & $16 \%$ & 1 & $5 \%$ & 0 & $0 \%$ & 0 & $0 \%$ & 4,74 & \\
\hline & 7 & 13 & $68 \%$ & 6 & $32 \%$ & 0 & $0 \%$ & 0 & $0 \%$ & 0 & $0 \%$ & 4,68 & \\
\hline & 8 & 11 & $58 \%$ & 7 & $31 \%$ & 2 & $11 \%$ & 0 & $0 \%$ & 0 & $0 \%$ & 4,53 & \\
\hline \multirow[t]{2}{*}{ Liderazgo } & 9 & 15 & $79 \%$ & 3 & $16 \%$ & 1 & $5 \%$ & 0 & $0 \%$ & 0 & $0 \%$ & 4,74 & \multirow[t]{2}{*}{4,62} \\
\hline & 10 & 14 & $74 \%$ & 4 & $21 \%$ & 0 & 0,00 & 0 & $0 \%$ & 1 & $5 \%$ & 4,58 & \\
\hline \multicolumn{14}{|l|}{ Promedio } \\
\hline de la & & & & & & & 4,62 & & & & & & \\
\hline
\end{tabular}

Fuente: Tomado de Paz et al. (2017).

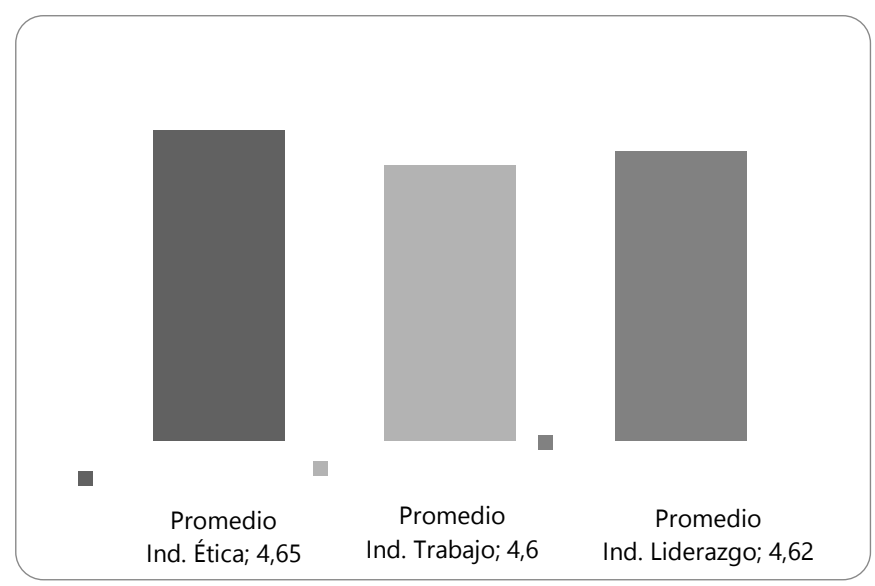

Figura 2. Factores de la responsabilidad social. Fuente: Adaptado de Paz et al. (2017)

del ítem 2 evidencia que el $79 \%$ de los encuestados manifestó que las universidades objeto de estudio siempre cuentan con trabajadores que actúan basados en la ética, mientras el $16 \%$ respondió casi siempre, y un $5 \%$, a veces. En el ítem 3 se observó que el $74 \%$ de la población objeto de estudio reveló que las universidades públicas siempre tienen políticas de contentivas a principios éticos para actuar de manera responsable consigo y el entorno, un $21 \%$ dijo que casi siempre, y un $5 \%$, casi nunca. El valor promedio del indicador es de 4,65, lo que muestra una muy alta presencia de los factores de responsabilidad social en el contexto de estudio.

Con respecto al ítem 4 del indicador Trabajo, se observa que el $58 \%$ de la población encuestada señaló que las universidades públicas siempre cuentan con empleados que desempeñan un trabajo colaborativo basado en principios de gestión de la ética social, mientras el $32 \%$ señaló la alternativa casi siempre y un $10 \%$, a veces.

Respecto del ítem 5, el $63 \%$ de los empleados dijo que las universidades públicas siempre tienen colaboradores 
que hacen su trabajo siguiendo la lógica de la dimensión científico-técnica; un $32 \%$ dijo casi siempre y el $5 \%$ restante indicó la alternativa a veces. Para el ítem 6 , los resultados muestran que el $79 \%$ de los encuestados afirmaron que los empleados siempre hacen su trabajo siguiendo la lógica de la dimensión ética, el $16 \%$ dijo casi siempre y el $5 \%$, a veces.

Para el ítem 7 se obtuvo como resultado que el $68 \%$ de los encuestados consideran que las universidades públicas siempre promocionan el trabajo ético en el cumplimiento de sus actividades de responsabilidad social, y un $32 \%$ dijo que casi siempre. Con respecto al promedio del indicador Trabajo, se obtuvo un valor de 4,60, equivalente a muy alta presencia.

De la misma manera, el ítem 8 referente al indicador Liderazgo, muestra que el $58 \%$ de los encuestados manifestaron que las universidades objeto de estudio siempre tienen líderes que cumplen un rol fundamental en el desempeño de la responsabilidad social institucional; el $31 \%$ indicó la alternativa casi siempre y un $11 \%$ a veces. El ítem 9 arrojó que el $79 \%$ de la población encuestada afirmó que las universidades públicas siempre cuentan con líderes que promueven el trabajo ético, el 16\% dijo casi siempre y un $5 \%$, a veces. Por otra parte, los resultados del ítem 10 evidencian que el $74 \%$ de los encuestados declararon que las universidades objeto de investigación siempre tienen líderes con una visión holística, que tiene en cuenta la riqueza de la vida humana, mientras el $21 \%$ dijo casi siempre y un $5 \%$, nunca. El promedio del indicador Liderazgo es de 4,62, equivalente a muy alta presencia de los factores de la responsabilidad social en el escenario universitario público.

Finalmente, los datos recogidos en la Tabla 2, que miden el comportamiento estadístico de la dimensión correspondiente a los factores integradores de la responsabilidad social en universidades públicas, alcanzaron una categoría de muy alta presencia, con un promedio aritmético de 4, 62 .

En consecuencia, se concluye que los factores de la responsabilidad social en las universidades públicas comprenden una relación para cultivar una actuación desde los principios éticos, transmisible a los colaboradores como parte del rol de líder para ejercer en la comunidad universitaria un desempeño responsable con su gente y el entorno de las comunidades adyacentes a ella; con el compromiso de ejercer su apoyo a favor del bienestar y la construcción de sociedades sostenibles.

\section{Discusión}

Según la información obtenida se muestra la discusión de los resultados en contraste con las teorías consultadas para analizar los factores de la responsabilidad social en las universidades públicas, se pudo observar, en relación al indicador Ética, que obtuvo un promedio de 4,65, lo que indica una alta presencia en el contexto estudiado, además permite evidenciar que las instituciones objeto de estudio desarrollan una dinámica institucional centrada en la ética, contando para el desempeño de la gestión institucional con colaboradores que ejecutan su actuación laboral contempladas en este factor de responsabilidad social.

Los resultados coinciden con el postulado teórico expuesto por Guédez (2006), según el cual la ética en el ámbito de responsabilidad social se evidencia en un elevado índice de compromiso, ya que no existe una responsabilidad social sin ética, aun si se realizan inversiones millonarias. En este sentido, la ética es el desarrollo propio y meramente subjetivo; en otro sentido, quiere decir que se desarrolla sin perjudicar a otras personas o instituciones. Por consiguiente, un comportamiento ético consiste no solo en evitar la corrupción, sino en respetar las libertades, los anhelos y las potencialidades de los demás, razón por la cual la ética es necesaria en las organizaciones.

El indicador Trabajo alcanzó un promedio de 4,60, ubicándose en la categoría alta presencia, lo que demuestra que las universidades públicas objeto de la investigación cuentan con colaboradores que desempeñan su trabajo basados en la responsabilidad social, siguiendo la lógica de la dimensión científico-técnica y ética. Estos resultados coinciden con Guédez (2008), quien sostiene que el trabajo es una actividad propia del ser humano, también otros seres actúan dirigiendo sus energías coordinadamente, aunado con una finalidad determinada. Sin embargo, el trabajo propiamente dicho, entendido como proceso entre la naturaleza y el hombre, es exclusivamente humano.

En relación con el indicador Liderazgo, este alcanzó un promedio de 4,60, lo que indica una alta presencia. Esto revela que las universidades públicas cuentan con líderes que juegan un papel fundamental en la responsabilidad social y promueven el trabajo ético con una visión holística. Estos resultados guardan relación con lo planteado por Guédez (2008), para quien el liderazgo juega un papel esencial en cada una de las dinámicas organizacionales, siendo un líder resonante quien crea más. El auténtico desarrollo del liderazgo debe asentarse en una visión holística que vaya más allá de una nueva planificación de carrera profesional y que tenga en cuenta toda la riqueza de la vida humana, tal como se evidencia en la gestión de las universidades públicas. 


\section{Conclusiones}

Tomando en consideración los resultados derivados de la presente investigación, cuyo propósito fue analizar la responsabilidad social como factor integrador en las universidades públicas, los resultados son los siguientes:

Se pudo observar, en relación con el indicador Ética, que las instituciones desarrollan una dinámica reflejada en la ética de sus colaboradores; al tiempo que actúan basándose en los principios éticos, así como políticas de responsabilidad social como parte de la voluntariedad que proporcionan identidad y cultura con los grupos de interés.

En cuanto al indicador Trabajo, se observa que las universidades públicas cuentan con colaboradores que desempeñan su labor basados en los principios de gestión de la responsabilidad social, siguiendo la lógica de la dimensión científico-técnica y ética para garantizar el sustento de un trabajo transparente consigo mismo, al igual que con otros de su entorno, como son las comunidades.

En relación con el indicador Liderazgo, se encontró que las universidades públicas tienen líderes que cumplen un rol fundamental en la puesta en práctica de la responsabilidad social institucional, promoviendo el trabajo ético con una visión holística que toma en cuenta toda la riqueza de la vida humana.

\section{Referencias}

Arias, F. (2012). El Proyecto de Investigación. Venezuela: Episteme.

Brittel, P. (2007). Lo que todo supervisor debe saber. México: Mac Graw-Hill.

Chávez, N. (2007). Introducción a la investigación educativa. Maracaibo: ARS Gráfica.

Chiavenato, I. (2011). Administración de recursos humanos. El capital Humano de las organizaciones. México: Mac Graw Hill.

Fernández, R. (2009). Administración de la responsabilidad social corporativa. España: Thompson.

Gallego, M. (enero-julio, 2006). La responsabilidad social de las organizaciones: ¿Factor de ventaja competitiva? Las acciones sociales de las organizaciones en relación con el tejido social. AD-MINISTER Universidad EAFIT, 8, 106-123.

García, E. \& González, I. (2015). La universidad educa para la libertad. Reflexiones de un humanismo personalista. I+ D Revista de Investigaciones, 5(1), 108123. Recuperado de https://doi.org/10.33304/revinv. v05n1-2015007

Global University Network for Education (2009).
Introducción en La Educación Superior en tiempos de cambio. Nuevas dinámicas para la responsabilidad social. Madrid: Mundi-Prensa.

Guanipa, M. (2010). Reflexiones básicas sobre investigación. Maracaibo: Universidad Rafael Belloso Chacín.

Guédez, V. (2006). Ética práctica de la Responsabilidad Social Empresarial. Venezuela: Planeta Venezolana.

Guédez, V. (2008). Ser Confiable. Responsabilidad Social y Reputación Empresarial. Colección Temas gerenciales. Venezuela: Planeta Venezolana. S. A.

Hernández, R., Fernández, C. \& Baptista, M. (2014). Metodología de la Investigación. México: Mc. Graw Hill.

Hurtado, J. (2008). Metodología de la Investigación. Venezuela: Magisterio.

Lussier, R. \& Achua, C. (2008). Liderazgo: Teoría, Aplicación y Desarrollo de Habilidades. (2a. ed.). México: Cengage Learning.

Méndez, C. (2012). Metodología Diseño y Desarrollo del Proceso de Investigación. Colombia: Limusa.

Núñez, M., Salom, J., Rosales, V. \& Paz, A. (2012). Responsabilidad Social Universitaria: enfoque de gestión ética compartida. Opción, 69, 579-594.

Paz, A., Harris, J. \& Molero, L. (2010). Ética, fundamento de la responsabilidad social universitaria. (Ponencia, II Jornadas Científicas Internas de la Universidad Dr. José Gregorio Hernández).

Paz, A., Núñez, G., Salom, J. \& Rosales, V. (2013). Responsabilidad social universitaria: moralidad o compromiso en la formación de valores éticos en la educación de futuro. Opción, 29(72), 97-116.

Paz, A., Paz, J. \& Franco, F. (2013). Responsabilidad social: una mirada sustentable hacia al ambiente en empresas mixtas petroleras. (Jornadas Científicas Nacionales Dr. José Gregorio Hernández "Encuentro de Saberes Universitarios"). Maracaibo, Venezuela.

Paz, A., Sánchez, J. \& Magdaniel, Y. (2016). Responsabilidad social. Un compromisodetodos. Venezuela:Universidad de La Guajira.

Paz, A., Sánchez, J., Magdaniel, Y., \& Robles, C. (2017). Estrategias de la responsabilidad social acción voluntaria en la universidad de la guajira. En Desarrollo Gerencial Revista de La Facultad de Ciencias Económicas Administrativas y Contables de La Universidad Simón Bolívar-Colombia, 9(1), 126-143.

Pelekais, C., \& Aguirre, R. (2008). Hacia una cultura de responsabilidad social. México: Pearson.

Pérez, F. (2009). La responsabilidad social universitaria. España: Consejo Social de la Universidad de Huelva. Recuperado de http://www.uhu.es/consejo.social/pdf/ documentos/responsabilidadsocialuniversitaria.pdf

Perozo, R. \& Paz, M. (2016). Estilos de liderazgo femenino como factor influyente en las habilidades de los equipos de trabajo en el sector Asegurador. Clío América, 10 (19), 8-22. 
Ramos, M. (2006). Valores y autoestima. Conociéndose a sí mismo en un mundo con otros. Venezuela: San Pablo.

Robbins, S. (2009). Comportamiento organizacional. (13a. ed.). Buenos Aires: Pearson.

Serrano, E. (2015). La relación entre el habitar-ethos y la ética. Antropología educativa. I+ D Revista de Investigaciones, 6(2), 6-18. Recuperado de https://doi. org/10.33304/revinv.v06n2-2015001

Soto, E. \& Cárdenas, J. (2007). Ética en las organizaciones. México: McGraw - Hill.

Tamayo, M. (2004). El Proceso de la Investigación Científica (4⿳a. ed.). México: Limusa.

Velásquez, M. (2008). Ética en los negocios, conceptos y casos (6a. ed.). México: Mac Graw-Hill. 